\title{
An unusual case of recurrent ventricular tachycardia
}

\author{
Ricardo Costa $^{1}$, Maria João Sousa ${ }^{1}$, and Severo Torres ${ }^{1}$ \\ ${ }^{1}$ Centro Hospitalar Universitário do Porto EPE
}

January 10, 2022

\begin{abstract}
Amiodarone is commonly used in acute care due to its effectiveness in several arrythmias and safety if structural heart disease is present or unknown. However, it can have a proarrhythmic effect. We presented a case of iatrogenic arrhythmic storm due to concomitant use of amiodarone plus tiapride resolved with isoproterenol.
\end{abstract}

An unusual case of recurrent ventricular tachycardia

Authors:

1. Ricardo Costa ${ }^{1}, \mathrm{MD}$, e-mail: ricardofcosta91@gmail.com

2. Maria João Sousa ${ }^{1}$, MD, e-mail: maria.sousa.101@sapo.pt

3. Severo Torres ${ }^{1}, \mathrm{MD}$, e-mail: severotorres@netcabo.pt

${ }^{1}$ Department of Cardiology, Centro Hospitalar Universitário do Porto, Largo Prof. Abel Salazar 4099-001 Porto, Portugal.

Corresponding author: Ricardo Costa; mobile phone: +351931783347; e-mail: ricardofcosta91@gmail.com; adress: Largo Prof. Abel Salazar 4099-001 Porto, Portugal

Declarations:

- Funding: none

- Conflicts of interest: none

- Ethical approval: The study was approved by the Ethics Committee of Centro Hospitalar Universitário do Porto

- Written informed consent was obtained from the patient to publish this report in accordance with the journal's patient consent policy

\section{Abstract}

Amiodarone is commonly used in acute care due to its effectiveness in several arrythmias and safety if structural heart disease is present or unknown. However, it can have a proarrhythmic effect. We presented a case of iatrogenic arrhythmic storm due to concomitant use of amiodarone plus tiapride resolved with isoproterenol.

Keywords: cardiac care, arrythmia; ECG, interpretation; iatrogenesis

\section{Case description}

A 49-year-old male with past medical history of alcoholic liver cirrhosis was admitted to the emergency room in hemorrhagic shock due to bleeding from esophageal varices, resolved by endoscopic treatment. Later, he developed atrial fibrillation and intravenous amiodarone was initiated. After that, he presented non-sustained polymorphic ventricular tachycardia. The electrocardiogram showed flattened $\mathrm{T}$ waves and 
slightly prolonged corrected QT interval (QTc) that was not noticed (Figure 1A). Magnesium sulphate and amiodarone (total dose of $2100 \mathrm{mg}$ in 36 hours) were administered to prevent ventricular dysrhythmia. Then, he developed recurrent sustained polymorphic ventricular tachycardia requiring defibrillation several times. The electrocardiogram revealed QTc higher than 700 milliseconds and T-wave alternans (Figure 1B). It was assumed iatrogenic QTc prolongation due to concomitant use of amiodarone plus tiapride. Isoproterenol with target heart rate of 100 beats per minute was initiated. Ventricular dysrhythmia terminated and QTc progressively normalized (Figure 1C).

Amiodarone is commonly used in acute care due to its effectiveness in several arrythmias and safety if structural heart disease is present or unknown [1]. However, it has proarrhythmic risk, especially in the presence of other conditions that prolong the QT interval [1]. Isoproterenol is a less invasive alternative to the temporary overdrive pacing in preventing ventricular dysrhythmia [2].

\section{Author Contributions}

Ricardo Costa was involved in the literature search and drafting of the manuscript. Maria João Sousa was involved in clinical care of the patient and revision of the manuscript. Severo Torres was involved in the revision of the manuscript.

\section{References}

1. Dan GA, Martinez-Rubio A, Agewall S, et al. Antiarrhythmic drugs-clinical use and clinical decision making: a consensus document from the European Heart Rhythm Association (EHRA) and European Society of Cardiology (ESC) Working Group on Cardiovascular Pharmacology, endorsed by the Heart Rhythm Society (HRS), Asia-Pacific Heart Rhythm Society (APHRS) and International Society of Cardiovascular Pharmacotherapy (ISCP). Europace. 2018;20(5):731-732an.

2. Al-Khatib SM, Stevenson WG, Ackerman MJ, et al. 2017 AHA/ACC/HRS Guideline for Management of Patients With Ventricular Arrhythmias and the Prevention of Sudden Cardiac Death: A Report of the American College of Cardiology/American Heart Association Task Force on Clinical Practice Guidelines and the Heart Rhythm Society. Circulation. 2018;138(13):e272-e391.

Figure 1A. Electrocardiogram performed between episodes of non-sustained polymorphic ventricular tachycardia: atrial fibrillation with controlled ventricular rate, flattened $\mathrm{T}$ waves and slightly prolonged corrected QT interval.

Figure 1B. Electrocardiogram performed between episodes of sustained polymorphic ventricular tachycardia: sinus rhythm, note the extreme corrected QT interval prolongation (more than 700 milliseconds) and the presence of $\mathrm{T}$-wave alternans (white stars).

Figure 1C. Electrocardiogram performed during drugs' washout; note the shorter QT interval. 

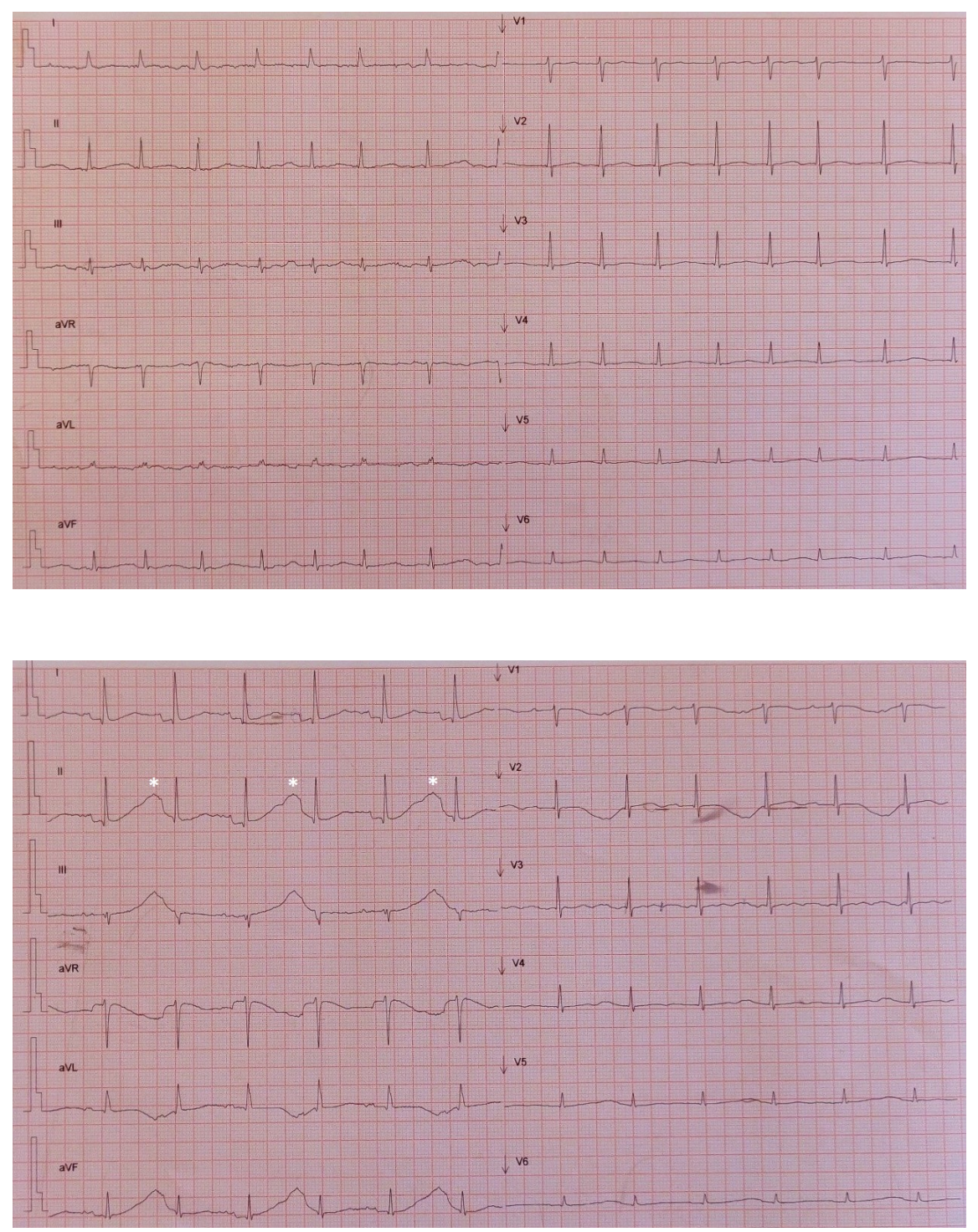


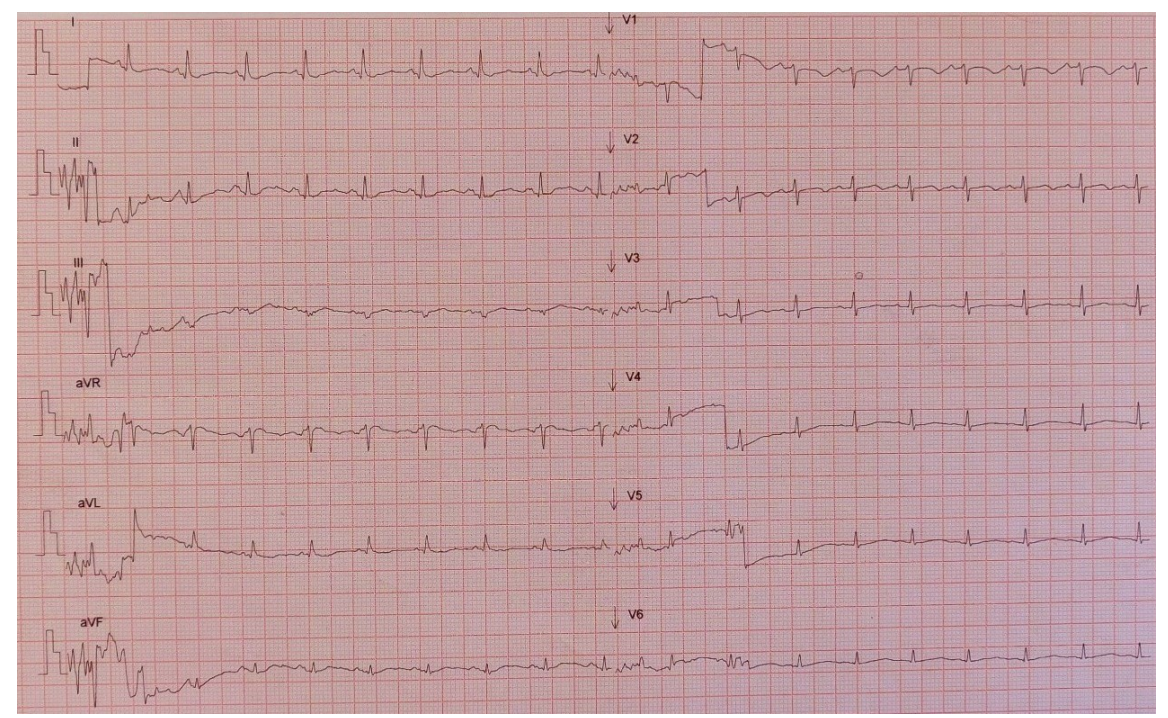

